\title{
Die Informatisierung des betrieblichen Erfahrungswissens und der „Imperialismus der instrumentellen Vernunft“"
}

\author{
Kritische Bemerkungen zur neotayloristischen Instrumentalismuskritik \\ und ein Interpretationsvorschlag aus arbeitssoziologischer Sicht
}

\section{Thomas Malsch}

Wissenschaftszentrum Berlin für Sozialforschung, Forschungsschwerpunkt Arbeitspolitik, Steinplatz 2, D-1000 Berlin

Zusammenfassung: Ein Grundthema soziologischer Theoriebildung, die Kritik der instrumentellen Vernunft, erhält durch die Informatisierung eine neue Virulenz. Die Computertechnologie, so wird befürchtet, werde die eigensinnigen Reflexions- und Kommunikationskulturen des gesellschaftlichen Lebens den Zwängen einer rücksichtslosen instrumentalistischen Rationalisierung unterwerfen. Der Realitätsbezug solcher Befürchtungen ist insbesondere hinsichtlich der Arbeitswelt kaum zu bestreiten, aber sie machen blind für eine Entwicklungsdialektik, die mit dem Theorem des „Imperialismus der instrumentellen Vernunft" (Weizenbaum) nicht entschlüsselt werden kann. Am Thema der informationstechnologischen Transformation von betrieblichem Erfahrungs- in Planungswissen möchte ich die These zur Diskussion stellen, daß der Computereinsatz in der industriellen Produktion zwar zur Einebnung und Formierung von Erfahrungswissen, Eigensinn und kommunikativer Kompetenz führt, gleichzeitig aber auf paradoxe Weise zu ihrer Erneuerung beiträgt. Dies ist darauf zurückzuführen, daß die Informatisierung des Erfahrungswissens im wachsenden Maße auch auf reflexive Informationskompetenz und "Selbstbeobachtung“ der Beschäftigten angewiesen ist.

Die Informatisierung stellt eine epochale Herausforderung für die industriekapitalistischen Gesellschaften dar. Ein ungewisser Horizont tut sich auf, Neues kündigt sich an, Altvertrautes gerät in Bewegung, Ängste und Hoffnungen werden geweckt. Technologieprojekte von gewaltigem Ausmaß und unübersehbarer Tragweite werden aufgelegt, politische Weichen gestellt, öffentlicher Streit über die Computertechnik flammt auf. Angesichts der raschen Ausbreitung der Mikroelektronik und der beschleunigten informationstechnologischen Durchdringung aller Lebensbereiche gerät auch die Soziologie unter Antwortdruck. Neue Begriffe wie „Informationsgesellschaft“ und „Computerkultur" machen die Runde. Diese sind sicherlich von nur vorläufigem oder gar zweifelhaftem Erklärungswert. Dennoch transportieren die neuen Begriffe und Interpretationsversuche richtungsweisende Intuitionen und Einsichten, an denen die Soziologie sich abarbeiten muß, um zu tragfähigeren Konzeptionen vorzustoßen. Das gilt auch für den Begriff des „Imperialismus der instrumentellen Vernunft", den der Informatiker J. Weizenbaum (1978) geprägt hat. Diesen Begriff möchte ich in meinem Beitrag zum kritischen Anlaß für einen weiterführenden Interpretationsvorschlag nehmen.
Das "Imperialismustheorem" Weizenbaums besagt, daß Informatik und Computertechnologie als Gestalten einer blindgewordenen naturwissenschaftlichen Zweckrationalität zu begreifen sind, deren "schleichendes Gift" (Weizenbaum 1978: 28) immer tiefer in unsere Lebens- und Arbeitswelt eindringe und alle nicht-instrumentellen Erlebens-, Ausdrucks- und Rationalitätsformen zerstöre. Mit seiner eindringlichen Warnung vor der Macht der Computer und der Ohnmacht menschlicher Vernunft reiht Weizenbaum sich ein in eine technikkritische Gesellschaftstheorie, die seit einigen Jahren immer energischer auftritt und die die Stichworte für eine einflußreiche populärwissenschaftliche Literatur liefert. Sie bietet sich all jenen als Plattform an, die in das affirmative Lob der "Informationsgesellschaft" nicht einstimmen und den Weg vom „stählernen Gehäuse“ Max Webers ins „elektronische Gehäuse“ Claus Kochs (1983) nicht mitgehen wollen. Ähnlich wie Weizenbaum brandmarkt Sherry Turkle (1984: 311) das imperialistische Bemühen einer rationalistisch halbierten Computerwissenschaft, „die intellektuellen Arenen anderer Disziplinen zu besetzen und zu kolonisieren“; Volpert (1985: 142) fordert, die Psychologie aus ihrem „Kolonialstatus“" zu befreien; Ortmann (1984: 99) sieht in der algorithmischen $\mathrm{Zu}$ richtung der gesellschaftlichen Realität einen „funktionalen Imperialismus“ am Werk; und auch 
Habermas (1981) spricht in seiner Theorie des kommunikativen Handelns von einer drohenden „Kolonialisierung der Lebenswelt“.

Die Ähnlichkeit der Begriffe kann natürlich nicht darüber hinwegtäuschen, daß es sich im einzelnen um recht unterschiedliche Interpretationsversuche handelt. Dennoch ist ihnen eine gemeinsame Grundauffassung zu eigen, die die Unterschiede jener Ansätze auf prononcierte Weise verblassen läßt. In dieser Grundauffassung wissen sie sich Horkheimers und Adornos „Dialektik der Aufklärung" (1947) verpflichtet. Es ist die Überzeugung, daß der von der europäischen Aufklärung entfesselte naturwissenschaftliche Rationalismus, einst als Befreiung aus mittelalterlichem Aberglauben gefeiert, längst in den modernen Aberglauben eines aggressiven Effizienzstrebens umgeschlagen sei. Dieses Effizienzstreben und das ihm entsprechende funktionalistische Denken werde durch die Computertechnologie auf die Spitze getrieben; das Bewußtsein der Differenz zwischen Mensch und Maschine drohe verloren zu gehen; unter einem immer dichteren Netz von Informations- und Kommunikationstechnologien werde das gesellschaftliche Leben ersticken; in beängstigende $\mathrm{Nä-}$ he sei die Schreckensvision der totalen Überwachung gerückt: „Foucaults Architektur von Gebäuden, die zwingende Blicke erlauben" wirke geradezu harmlos angesichts des unaufhaltsamen Aufstiegs einer „Architektur von Computern und Programmen, die zwingendes Wissen ermöglichen" (Ortmann 1984: 194). In der Arbeitswelt werde der Eigensinn der Arbeitskraft schon heute durch die berüchtigten Personalinformationssysteme (PIS) und Betriebsdatenerfassungssysteme (BDE) eingegrenzt, bis in seine feinsten und intimsten Verästelungen hinein ausgespäht und immer perfekterer informationstechnologischer Kontrolle und Formierung unterworfen.

Zweifellos spielt die Arbeitswelt eine Vorreiterrolle auf dem Wege in die "Informationsgesellschaft", denn hier ist der Computereinsatz besonders weit vorangetrieben worden. Hier kann sich das Imperialismustheorem auf ein Zukunftsszenario von unbestreitbarem Realitätsbezug berufen: Werkhallen und Büros werden informationstechnologisch miteinander vernetzt; alle Unternehmensbereiche von der Konstruktion bis zum Vertrieb, von der Produktion bis zur Personalplanung werden in einem rechnerintegrierten Verbundsystem zusammengeschlossen; Steuerungs- und Informationssysteme werden zu einem immer mächtigeren Kontrollapparat ausgebaut, der jede einzelne Handlung und jede Unterlassung der Arbeitnehmer einer lückenlosen Überwachung unterwirft. Die computergestützten Verfahren der Gewinnung und Verarbeitung von Informationen könnten die alten tayloristischen Methoden des Arbeitsstudiums und der Wissensenteignung ins Extrem übersteigern. Das mühsame Einsammeln jener überlieferten Faustregeln und Fachkenntnisse, die Taylor als "größtes Gut eines jeden Handwerkers" bezeichnet hatte, werden qua Informationstechnologie auf eine qualitativ andere Grundlage mit mühelosen Steigerungsmöglichkeiten gestellt. Auf informationstechnologischer Grundlage könnten sich der Wissensenteignung und der Transformation von Personal- und Produktionsdaten in Managementwissen neue Dimensionen einer bisher unerreichbaren Formierung und Überwachung der Beschäftigten eröffnen. Volpert (1985: 28) spricht deshalb von einer „neuen Taylorisierung“, die ,ihre wesentliche Stütze in der Computertechnologie" habe.

In der industriesoziologischen Diskussion hat das Imperialismustheorem während der letzten Jahre an Überzeugungskraft eingebüßt. Entsprechende neotayloristische Kontroll- und Deprivationsthesen, wie sie Ende der 70er Jahre im Anschluß an Braverman (1978) oder Sohn-Rethel (1970) entwickelt wurden (vgl. Institut für Sozialforschung 1981), befinden sich auf dem Rückzug oder sind in einen Gährungsproze $\beta$ übergegangen, der noch andauert. Das ist nicht zuletzt ein Ergebnis empirischer Forschungserfahrungen, die zu einem differenzierteren Bild der Computertechnologie beigetragen haben (zusammenfassend vgl. Sorge 1985; Veltz 1986; Mill 1986; Malsch/Seltz 1987). Danach hat der Computereinsatz keineswegs einheitliche Folgen für die industrielle Qualifikations- und Belastungsentwicklung; Abstraktifizierung und Intellektualisierung der Arbeit scheinen durchaus vereinbar zu sein; Arbeitspolitik und betriebliche Herrschaftsbeziehungen komplizieren sich, allerdings bei strukturell unveränderter Machtasymmetrie zugunsten des Managements; Anzeichen für eine totale Subsumtion der lohnabhängig Beschäftigten sind dagegen nicht zu beobachten. Solche Befunde lassen sich mit den begrifflichen Mitteln des Imperialismustheorems nicht oder bestenfalls als Überbleibsel aus einer vorinformatischen Industrialisierungsstufe deuten. Hier ist eine sozialwissenschaftliche Erklärung gefordert, der es zu zeigen gelingt, wie jene empirischen Phänomene, an denen das Imperialismustheorem scheitert. sich auf der Grundlage informationstechnologischer Rationalisierung trotz fortschreitender Wissensenteignung und Abstraktifizierung reproduzie- 
ren können. Dies werde ich am Thema des betrieblichen Erfahrungswissens zu begründen versuchen, indem ich den gesellschaftstheoretischen Anspruch beherzige, den die Vertreter des Imperialismustheorems längst aufgegeben haben: den Anspruch, die Informatisierung als dialektische Entwicklung zu dechiffrieren.

Vorwegnehmend läßt sich der im weiteren auszuführende Gedankengang wie folgt umreißen: Im Zuge der Informatisierung wird die systematische Gewinnung und Verarbeitung von Erfahrungswissen für den betrieblichen Innovations- und Rationalisierungsproze $B$ immer bedeutsamer. Dabei stößt der auf „Fremdbeobachtung“ beruhende tayloristische Modus der Wissensgewinnung an seine Grenzen. Er muß durch einen neuen informationstechnologischen Modus der Enteignung, Erzeugung und Transformation des Erfahrungswissens ergänzt werden. Dieser ist auf ,Selbstbeobachtung“, aktive Informationskompetenz, intelligente Dateneingabe und den eigenständigen Algorithmisierungsbeitrag der betroffenen Fachkräfte angewiesen. Selbstbeobachtung ist reflexiv auf die Erfahrung der Diskrepanz gerichtet, die sich zwischen Realprozeß und Computerprogramm auftut. Sie wird auf informationstechnologischer Grundlage beständig reproduziert und befördert somit instrumentalistische Denkformen und den kritischen Umgang mit ihnen gleichermaßen. Die auf Selbstbeobachtung angewiesene Informationskompetenz gibt dem Personal zudem ein neuartiges Drohpotential der Datenmanipulation und der Wissenszurückhaltung an die Hand, das im spannungsreichen Nebeneinander von Fremdbeobachtung und Selbstbeobachtung virulent werden könnte. Diese Spannungen sowie weitere Konfliktlinien und Friktionszonen begünstigen eher reflexives und kommunikatives Denken statt eines eindimensionalen Instrumentalismus. Sie sind strukturell eingebaut in einen kreislaufförmigen Prozeß der Wissenstransformation, der vom Arbeitsvermögen ausgeht und zu diesem zurückkehrt. Im Transformationsprozeß der Gewinnung, Objektivierung und Rückkehr des Wissens wird das Erfahrungswissen zugleich destruiert und erneuert. In diesem Doppeleffekt scheint mir die Gewähr zu liegen, $\mathrm{da} B$ die Dialektik von instrumenteller und reflexivkommunikativer Rationalität nicht zum Stillstand kommt. Die Informatisierung vollzieht sich mithin nicht als eindimensionale, sondern als widersprüchliche Entwicklung. Zwar ebnet sie die Vielfalt betrieblicher Kommunikationskulturen ein und richtet das Arbeitsvermögen gemä $\beta$ der instrumentalistischen Logik der Computersysteme zu; dennoch emeuert sie auf paradoxe Weise das Erfahrungswissen, das eigensinnige Reflexionsvermögen und die kommunikative Kompetenz der Arbeitskraft und hält die nicht-instrumentellen Dimensionen gesellschaftlicher Lebensmöglichkeiten offen.

\section{II}

In der Vorstellungswelt des (neo-)tayloristischen Kontrolltheorems spielt der Gedanke der Wissensenteignung eine zentrale Rolle. Danach ist Wissensenteignung zunächst durchaus wörtlich zu nehmen: Unter der Regie des Betriebsmanagements wird das Fachwissen des qualifizierten Handwerkers durch fremde Spezialisten ausgeforscht, seine Berufsgeheimnisse werden ihm abgeluchst, sie werden systematisch analysiert und in stark vereinfachte Arbeitsmethoden umgesetzt, nach denen der Handwerker sich fortan zu richten hat. Damit werden Geschicklichkeit und Virtuosität des Handwerkers nicht mehr gebraucht, liegen brach und verkümmern. So kommt eine Entwicklung in Gang, in der das traditionelle Handwerk rücksichtslos entwertet wird. Braverman (1978) hat diese Entwicklung sorgfältig untersucht und in seiner Studie zur „Degradation der Arbeit im 20. Jahrhundert" eindringlich beschrieben. Für ihn sind die äußerst beschränkten Fähigkeiten des modernen Massenarbeiters nichts anderes als die traurigen Überreste der einstmals so stolzen frühindustriellen Handwerkerkompetenz. Sie sind das Ergebnis einer historischen Realentwicklung, einer langsam aber stetig sich durchsetzenden Tendenz zur Dequalifizierung, Entsinnlichung, Entwertung und Kontrolle lebendiger Arbeit, die nach Bravermanscher Lesart durch die Computertechnologien vollendet werde. Nimmt man die Aussage Bravermans und etlicher anderer Autoren (Briefs 1980; Ortmann 1984; Volpert 1985; Schmiede/von Greiff 1985) beim Wort, so käme die säkulare Degradierung der gesellschaftlichen Arbeit mit der absoluten Wissensenteignung zu ihrem folgerichtigen AbschluB: Dem totalen Wissensmonopol stünde das nackte Unwissen eines auf abstrakte Funktionserfüllung reduzierten Arbeitsvermögens gegenüber, bei dem es buchstäblich kein Wissensquäntchen mehr zu holen gäbe.

Gegenüber dieser Interpretationsfigur, die in der Informatisierung lediglich die modernste Steigerungsform eines eindimensionalen Rationalismus zu sehen vermag, lassen sich Einwände vorbringen. Einmal sind die Befunde einer ,ursprüngli- 
chen" Wissensenteignung aus der Übergangsperiode vom frühindustriellen Handwerk zur großindustriellen Massenproduktion nicht vorschnell zu verallgemeinern und auf spätere Perioden der industriekapitalistischen Rationalisierung ahistorisch zu projizieren. Außerdem ist die Vorstellung einer absoluten Wissensenteignung empirisch unhaltbar, denn selbst das äußerst beschränkte Erfahrungswissen des repetitiven Fließbandarbeiters enthält noch Optimierungsschätze, die sich im tagtäglichen Arbeitshandeln ansammeln und die von den Arbeitskräften zu ihrer Arbeitserleichterung und Entlastung ebenso wie zur inoffiziellen Methodenverbesserung genutzt werden. Dieser Einwand läßt sich freilich auch als Bestätigung der Bravermanschen Verelendungstheorie interpretieren.

Die Annahme nämlich, daß ein Restwissen als „tacit knowledge“ immer übrig bleibt, an das das Management nicht herankommt (Polanyi 1985; Wood 1986; Karlsen/Oppen 1986), bleibt dem neotayloristischen Enteignungsszenario auf merkwürdig defensive Weise verhaftet. Es ist ein Einwand, der die Dominanz der Wissensenteignung prinzipiell bestätigt, wenngleich er auf nicht unsympatische Weise die Widerstandschancen eines lebendigen Erfahrungswissens, sich in den Nischen der Organisation festzukrallen, hervorhebt. Es handelt sich also nicht um eine grundsätzliche Kritik, sondern eher um eine empirisch modifizierte Betrachtungsweise, die den Boden des Imperialismustheorems nicht verläßt.

Um sich aus der tayloristischen Befangenheit der sozialwissenschaftlichen Kritik an Taylors Ideen vom „scientific management“" zu lösen, ist ein Perspektivwechsel nötig, der sich auf folgende Frage richtet: Wie kommt es, daß es für die professionellen Wissensenteigner des Managements offenbar immer wieder etwas zu holen gibt, daß sich das Erfahrungswissen der lohnabhängig Beschäftigten stets aufs Neue zu reproduzieren scheint? Mit Hack/Hack (1985: 569) läßt sich die Erneuerung des Erfahrungswissens als Folge der technologischen Entwicklung beschreiben. Neuen Produktionstechnologien korrespondiert neues Erfahrungswissen. Denn.die produktive Nutzung neuer Maschinerie und neuer Herstellungsverfahren, in welchen altes Erfahrungswissen objektiviert ist, muß begreiflicherweise neu gelernt werden. Dies wird in der Anlaufphase neuer Produktionsanlagen, in der zumeist die erfahrensten Fachkräfte herangezogen werden, besonders augenfällig. Gegen Braverman argumentieren die beiden Autoren daher völlig zu Recht, daß eines der ,wirklich erwähnenswerten Defizite“ seiner „,ansonsten ungeheuer eindrucksvollen Arbeit . . . in der völligen Ausblendung dieser Gegenbewegung der ,Wiederaneignung" des gesellschaftlich Objektivierten“ bestehe.

Das Interessante an dieser Überlegung ist natürlich nicht in der an sich trivialen Beobachtung neuer Qualifikationsanforderungen bei neuen Produktionstechnologien zu sehen. Interessant ist vielmehr, daß Hack/Hack diesen Sachverhalt im Kontext der Transformation und Objektivation von Wissen interpretieren. Hier liegt meines Erachtens der Schlüssel zum Verständnis des Zusammenhangs zwischen Enteignung und Wiederaneignung von industriellem Erfahrungswissen. Diesen Gedanken werde ich am Transformationsproze 3 von Erfahrungswissen in Planungswissen (Malsch 1983) zu entwickeln versuchen. Geht man dem Transformationsproze $\beta$ des Erfahrungswissens genauer nach, so zeigt sich, daß Braverman vom Begriff der Wissensenteignung letztlich doch nur metaphorischen Gebrauch gemacht und ihn zu einem Kampfbegriff ohne analytische Aussagekraft degradiert hat.

\section{III}

Die Transformation von Erfahrungswissen in Planungswissen läßt sich als Kreislaufbewegung darstellen, in welcher personengebundene Kenntnisse weder spurlos verlöschen noch vollends im Planungswissen aufgehen, wie es von den Enteignungs- und Kontrollthesen des Imperialismustheorems nahegelegt wird. Die Wissenstransformation durchläuft drei analytisch unterscheidbare Phasen. In der ersten Kreislaufphase wird das Erfahrungswissen unter selektiven Gesichtspunkten empirisch erhoben, beobachtet oder abgefragt und schriftlich fixiert oder elektronisch gespeichert: Wissensgewinnung. Es existiert danach auf zweifache Weise, nämlich im Arbeiterkopf und in schriftlicher oder elektronischer Vergegenständlichung. In der zweiten Phase wird das abgespeicherte Erfahrungswissen systematisch entfaltet und in kontextfreies Planungswissen transformiert: Wissensobjektivation oder Verwissenschaftlichung. Es existiert nunmehr in kontextfreier, abstraktifizierter Gestalt und seine Herkunft ist ihm nicht mehr anzusehen. In der abschließenden dritten Phase wird das objektivierte Wissen in Anwendungswissen übersetzt und kehrt in den Produktionsprozeß zurück. Hier nimmt es die Gestalt von Maschinerie, Organisation und Planung an und tritt dem Erfahrungswis- 
sen der lohnabhängigen Produzenten destruierend und erneuernd gegenüber: Wissensrückkehr.

Der Transformationsprozeß von Erfahrungs- in Planungswissen ist also kein endlicher Vorgang, der mit der absoluten Entleerung der Arbeiterköpfe endet oder nach einer Periode der ursprünglichen Wissensenteignung des frühindustriellen Handwerks zum Stillstand kommt. Vielmehr handelt es sich um eine zyklische Bewegung, die mit der Rückkehr zu ihrem Ausgangspunkt neu beginnt. Der Gedanke der zyklischen Wiederholung von Erzeugung, Objektivation und Rückkehr des Wissens schließt formell die Möglichkeiten erweiterter ebenso wie verengter Wissensreproduktion ein; die Möglichkeiten der Substitution, Entwertung und Destruktion von altem Erfahrungswissen ebenso wie die Herausbildung von neuem Erfahrungswissen. Kontinuierliche Erneuerung des Kreislaufprozesses impliziert ferner, daß sich Gewinnung, Verwissenschaftlichung und Rückkehr des Wissens gleichzeitig vollziehen. Umso wichtiger ist es, die drei Phasen als distinkte Vorgänge zu begreifen, die ihr relatives Eigenleben führen und sich gegeneinander verselbständigen können. Das eröffnet eine Untersuchungsperspektive, die den Blick auf reproduktive Störungen und Friktionen im Übergang von einer Wissensform zur nächsten freigibt. Solche Störungen sind nicht als behebbare Fehler eines (noch) imperfekt modellierten kybernetischen Regelkreises zu verstehen, sondern als Widersprüche der Kapitalverwertung, die sich auf je unterschiedlicher Entwicklungsstufe der gesellschaftlichen Arbeit aufs Neue herausbilden, auflösen und rekonturieren. In diesem Sinne läßt sich die Informatisierung als neue Entwicklungsstufe gesellschaftlicher Arbeit beschreiben.

Die Informatisierung des Erfahrungswissen ist ein neuer Modus der Wissensobjektivation, in welchem sich Informationsgewinnung und Wissenserzeugung im großindustriellen Maßstab herausbilden. Information wird nicht mehr nur punktuell, sondern flächendeckend erzeugt. Um den „Informationsrohstoff" in der ersten Phase des Wissenskreislaufs, gewissermaßen an seiner Quelle, systemgerecht abnehmen zu können, wird eine algorithmische Durchdringung des Erfahrungswissens und seines Entstehungszusammenhangs notwendig. Diese ist weitaus voraussetzungsreicher als das systematische Arbeitsstudium Taylors oder als die Erzeugung experimenteller Massendaten in den "Science Based Industries“. Der informationstechnologische Prozeß der Wissenserzeugung gewinnt an Breite, indem mehr und mehr Beschäftigten- gruppen an die Terminals der Informationssysteme gesetzt und indem mehr und mehr Bereiche ihres Wissens und Könnens abgefragt werden. Er gewinnt an Tempo, indem die Reservate des Erfahrungswissens, die noch nicht vermessen, kartographiert und an die „zivilisatorischen“ Infrastrukturen der Computersysteme angeschlossen worden sind, immer schneller zusammenschrumpfen. Und er gewinnt an Systematik, indem die Ideen der Arbeitnehmer immer zielsicherer für den Innovations- und Rationalisierungskreislauf erschlossen werden.

Im Zuge dieser „Finalisierung“ (Böhme et al. 1972) des Erfahrungswissens kommt es zu einer entscheidenden Differenzierung im Modus der Wissensgewinnung: Informationsgewinnung durch „Fremdbeitrag“ und durch „Eigenbeitrag“. Der tayloristische Modus der Wissensgewinnung beruht auf Beobachtungs- und Erhebungstechniken, die von einem außenstehenden Spezialisten auf die Arbeitsverausgabung der Beschäftigten angewandt werden: Fremdbeobachtung. Diese verzichtet systematisch auf eine aktive Unterstützung oder Mithilfe durch die Betroffenen. Personalinformationssysteme verlängern und potenzieren auf dieser Linie der Fremdbeobachtung die Kontroll- und Interventionschancen des Managements, indem sie den Informationszugriff auf die Person des Arbeitsvermögens ausdehnen. Dies reicht bei zunehmender Informatisierung nicht mehr aus. Deshalb bildet sich eine neuartige Informationskompetenz der Beschäftigten heraus, die den intelligenten Eigenbeitrag des Arbeitsvermögens ins Zentrum der Informationsgewinnung rückt. Die Objektivation des Erfahrungswissens ist in wachsendem Maße auf die subjektive Interpretations- und Informationsleistung angewiesen: Selbstbeobachtung.

Hack und Hack (1985: 568) unterschätzen die Differenz zwischen Fremdbeobachtung und Selbstbeobachtung, wenn sie davon sprechen, daß von der „wissenschaftlichen Betriebsführung“ Taylors bis hin zu den neuen Methoden der "Selbstaufschreibung“ bei geistiger Arbeit „eine funktional recht gradlinige Entwicklung“ zur Erstellung immer neuer Objektivationsmöglichkeiten „des je bei den Arbeitskräften situierten Wissens und Könnens“ verlaufe. Denn die neuen Methoden der "Selbstaufschreibung“ indizieren einen Bruch mit dem tayloristischen Enteignungsmodus, der mit der Informatisierung erst richtig zum Zuge kommt, freilich ohne die tayloristische Fremdbeobachtung restlos zu ersetzen. Vielmehr kristallisiert sich ein konfliktreiches Nebeneinander der beiden Modi 
der Wissenserzeugung heraus. Denn auch die Fremdbeobachtung wird auf eine informationstechnologische Grundlage umgestellt und verliert keineswegs ihre Wirksamkeit. So entsteht eine hochsensible Konfliktzone, in der sich die Beschäftigten unter das Damoklesschwert ihres „Datenschattens" (Myrell 1984) gestellt sehen und gleichzeitig aufgrund ihrer neuartigen Informationskompetenzen mit wachsenden Gegenmachtchancen ausgerüstet werden.

Weitere Konfliktlinien kommen hinzu: Unter Beibehaltung konventioneller arbeitskraftbezogener Ökonomisierungsziele entsteht in der dritten Kreislaufphase der Wissensrückkehr in den Produktionsprozeß ein Kompatibilitätsproblem zwischen Arbeitsintensivierung durch restriktive Planvorgaben und Ausführungsvorschriften sowie erweiterten Handlungs- und Entscheidungskompetenzen im Umgang mit hypertrophen Hard- und Softwaresystemen. Die neuen Informationskompetenzen führen wiederum zu neuem Planungswis sen, das in Konzepte des intensivierten und verengten Arbeitseinsatzes umgesetzt wird. Dadurch baut sich ein Gegensatz zwischen erweiterten Handlungs-, Kommunikations- und Ermessenserfordernissen in der ersten und restringierten Handlungskompetenzen in der dritten Kreislaufphase auf (Malsch 1983: 243ff.).

Hier deutet sich ein komplexes Konglomerat von Kompatibilitätsproblemen und Widerspruchskonstellationen an, das Analysemöglichkeiten für diverse empirische Problemlagen, Konfliktfelder, Kompromiß- und Regelungschancen eröffnet. Diese widersprüchliche Gemengelage macht es unwahrscheinlich, daß die Informatisierung in ,totaler Systemintegration“ (Kubicek/Rolf 1985: 29) endet. Demgegenüber werde ich am Problem der auf "Selbstbeobachtung“ angewiesenen Informationskompetenz nun konkret aufzuweisen versuchen, warum und wie die Entwicklungswidersprüche der Informatisierung eine kommunikativ-reflexive Rationalisierung in Gang setzen können.

\section{IV}

Auf allen Ebenen des betrieblichen Produktionsprozesses und selbst im streng deterministischen Arbeitsvollzug findet laufend ein mündlicher Informationsaustausch statt, dessen unverzichtbare Bedeutung den Beteiligten kaum bewußt ist, obgleich ohne ihn nichts geht. Dieser mündliche Informationsaustausch wird durch schriftliche Kommunikation ergänzt oder substituiert, schriftliche
Kommunikationsformen werden formalisiert und standardisiert und schließlich von elektronischen Medien übernommen. Das hat tiefgreifende Folgen für die Arbeits- und Kommunikationsleistung der Beschäftigten. Auf informatisierter Grundlage werden die vielschichtigen Beobachtungs-, Interpretations- und Mitteilungsleistungen der Arbeitskraft auf eine computergemäße Informationskompetenz umgestellt.

So wird die Strichliste des Qualitätskontrolleurs durch einen EDV-gerechten Fehlercode abgelöst; der Instandhalter liest die Vollzugsmeldung seines Reparaturauftrags am Terminal ein; und der Facharbeiter im Maschinenbau hält die Fertigungssteuerungsabteilung über den täglichen Zeitfortschritt seines Fertigungsauftrags per EDV auf dem laufenden. Damit rückt der intelligente Umgang mit abstrakten Symbolen und Verknüpfungsregeln ins Zentrum eines systematischen Erzeugungsprozesses von Planungswissen, der gegenüber vorinformatischen Objektivierungsprozessen ein neues Niveau der „Abstraktifizierung“ (Schmiede 1983) anzeigt. Denn der Umgang mit der EDV bleibt den arbeitenden Subjekten nicht äußerlich, sondern er wirkt strukturierend, standardisierend und abstraktifizierend auf ihre informationsgenerierenden Wahrnehmungen und Beobachtungen zurück. Hier werden in der Tat jene Denkformen befördert, vor denen die Theoretiker des Imperialismustheorems warnen.

Das ist freilich nur die halbe Wahrheit der Informatisierung des betrieblichen Erfahrungswissens. Es gibt nämlich unübersehbare Anzeichen für einen reflexiven Umgang mit der binären Logik der Computertechnologien, der gleichsam immanent aus ihren Implementations- und Nutzungsformen hervorgeht. Der Schlüssel zum Verständnis des nicht-instrumentalistischen Umgangs mit mikroelektronischen Informations- und Steuerungssystemen liegt in der wachsenden Bedeutung von "Selbstbeobachtung". Auf informationstechnologischer Grundlage differenziert sich in der ersten Kreislaufphase der Wissenstransformation der subjektive Eigenbeitrag zur Informationserzeugung als spezifische Arbeitsaufgabe heraus.

Die Beispiele des Qualitätskontrolleurs, des Instandhalters und des Maschinenbaufacharbeiters machen dies deutlich. Hier ist eine subjektive Informationsleistung gefordert, die den betreffenden Arbeitskräften niemand abnehmen kann. „Fremdbeobachtung" ist hier nahezu ausgeschlossen. Relevante Ereignisse können nicht am fertigen Resultat erhoben werden, da sie nicht antizipierbar sind, 
nur im unmittelbaren Arbeitsvollzug wahrgenommen werden können und nach Arbeitsende verschwunden bzw. bloß noch im Gedächtnis der Arbeitskraft vorhanden sind. Nur der Arbeitende selbst kann die von ihm wahrgenommenen oder herbeigeführten Ereignisse in speicherbare und prozessierbare Informationen umsetzen. Das ausführende Personal wird deshalb zunehmend als Rohstofflieferant von Primärinformationen interessant. Die informatisierte Wissenserzeugung benötigt nämlich ein immer breiteres und spezifizierteres Spektrum an Primärinformationen, die in erster Instanz von den Arbeitskräften der ausführenden Ebene selbst produziert werden. Bei wachsendem Informationsbedarf sind computergestützte Informations- und Steuerungssysteme mehr denn je auf deren korrekte, intelligente und prompte Dateneingabe angewiesen.

Was passiert, falls der benötigte Informationsrohstoff doch fehlerhaft ist, sich verzögert oder ganz ausbleibt, das ist in der einschlägigen Literatur am Beispiel des „Vorderwassers" im Maschinenbau (Manske/Wobbe 1984; Ortmann 1984) ausgiebig diskutiert worden: Um ihren Dispositionsspielraum zu erweitern und sich gegen Auftragsschwankungen abzusichern, horten die Facharbeiter stets eine gewisse Reserve an Akkordscheinen, die sie erst mit zeitlicher Verzögerung verrechnen - sie bilden Vorderwasser. Vorderwasser ist bei konventioneller Auftragssteuerung durchaus funktional, hat aber fatale Konsequenzen für rechnergestützte Produktionsplanungs- und -steuerungssystems (PPS), die bei zeitlich verzögerter Auftragsabrechnung im Wolkenkuckucksheim operieren müßten. Die Reihe ähnlich gelagerter Beispiele läßt sich nahezu beliebig verlängern. Stets dreht es sich um die Kardinalfrage der Primärdatenqualität und ihrer zeit- und sachgerechten Eingabe.

Die Beschäftigten, die hinsichtlich der Informationsgewinnung einen Kompetenzzuwachs verbuchen können, sehen sich allerdings hinsichtlich der ..Informationsrückkehr" neuen Planungs- und Steuerungsmethoden sowie neuen Kalkulationstechniken des Ressourcen- und Personalbedarfs gegenüber, die ihre Dispositionsspielräume bei der Arbeitsausführung tendenziell einengen und ihre Arbeitsverausgabung intensivieren. Die rechnergestützte Instandhaltungsplanung beispielsweise nimmt den Fachkräften wichtige Dispositionschancen, sobald sie das Planungssystem mit einer entsprechenden Wissensgrundlage versorgt haben. Wartungsintervalle werden nicht mehr wie bisher nach Faustregeln und Absprachen zwischen Ferti- gungs- und Instandhaltungspersonal festgesetzt, sondern automatisch berechnet und vorgegeben. Inspektions- und Reparaturpläne werden per EDV-Programm erlassen. Sobald das Management dieses Rationalisierungspotential auszureizen beginnt, verliert das Instandhaltungspersonal wichtige dispositive Kompetenzen hinsichtlich der Arbeitsausführung. Es kommt zu einem Widerspruch zwischen Kompetenzverlust bei der Arbeitsausführung und Kompetenzgewinn bei der Informationserzeugung. Allgemeiner ausgedrückt: In der ersten Kreislaufphase der Wissenstransformation werden dem Personal gesteigerte Informationskompetenzen der intelligenten Dateneingabe abverlangt, die in der dritten Phase als restringierende Planvorgaben zurückkehren und den Handlungsspielraum des Personals einengen. Derartige Paradoxierungen einer gleichzeitigen Entfaltung und Restringierung von Entscheidungsautonomie sind in die Logik des Transformationskreislaufs von Erfahrungswissen und Planungswissen strukturell eingebaut.

Dieser Widerspruch zwischen Kompetenzgewinn bei der Informationserzeugung und Kompetenzverlust bei der Informationsumsetzung in Arbeitshandeln ließe sich möglicherweise überwinden, wenn es dem Mangement gelänge, den subjektiven Eigenbeitrag der Beschäftigten bei der Informationsgewinnung auszuschalten. Soweit ich sehe bieten sich hier prinzipiell zwei Wege an. Der erste Weg wäre der einer Arbeitsteilung, die zwischen die Erzeugungsquelle von Informationen und deren Ėingabe ins System einen oder mehrere Zwischenträger schaltet und damit die direkten Manipulationschancen der unmittelbar Betroffenen mindert. Dieser Weg von eingebauten Zwischenkontrollen ist aufwendig und kostspielig. Er potenziert letztlich nur das Störpotential, da es sich auch bei den nur mittelbar betroffenen Zwischenträgern um menschliche „Störquellen“ handelt.

Der zweite und möglicherweise aussichtsreichere Weg wäre der einer Automatisierung der Primärdatenerzeugung. So ist es bei hochautomatisierten Produktionsprozessen schon heute möglich, relevante Prozeßdaten und Störungsinformationen vollautomatisch zu erzeugen. Dies könnte zukünftig auch für jene Informationen über Systemzustände und Korrekturmaßnahmen gelten, die bislang manuell ausgeführt und per Selbstbeobachtung erhoben werden. Auf diesem Wege könnte das Drohpotential der Wissenszurückhaltung und Datenmanipulation ausgetrocknet und dauerhaft entschärft werden (Malsch 1983: 249). Die Frage, 
die sich dann stellt, ist die, ob sich unter den Bedingungen einer weitgehenden Automatisierung der Informationserzeugung die negative Utopie der Totalkontrolle nicht doch noch verwirklichen ließe.

Jazbinsek (1986) hat diese Frage am Beispiel der Meßwartentätigkeit zu beantworten versucht. Auf den Bildschirmen des Leitstandes bzw. der Meßwarte einer verfahrenstechnischen Großanlage werden die Prozeßdaten vom Steuerungsrechner automatisch erzeugt. Geht man nun vom Idealtypus einer lückenlos automatisierten Datenerzeugung bei perfektionierter Sensortechnik aus, so sind damit die für den Handlungsspielraum und für das Erfahrungswissen des Meßwarenpersonals prekärsten Prämissen benannt. Selbst unter diesen Prämissen verschwinden die Entscheidungsspielräume der Meßwarenarbeiter jedoch keineswegs. Jazbinsek zufolge kommt es unter den idealtypischen Bedingungen der automatischen Erfassung aller relevanten Prozeßinformationen nämlich zu einer eigentümlichen Problemverschiebung. „Problematisch ist dann nicht, ob alle relevanten Informationen technisch zu erfassen sind, sondern ob alle technisch erfaßten Informationen relevant sind (...). Die Frage nach dem Entscheidungsspielraum des Steuerungspersonals muß deshalb in die Frage umformuliert werden, inwieweit sich der Informationsbedarf ex ante bestimmen, also vorweg entscheiden läßt." (Jazbinsek 1986: 12)

Dies scheint nun aber ausgeschlossen zu sein. Auch wenn die Mensch-Maschine-Kommunikation problemlos funktionieren sollte, bleibt die Meßwartentätigkeit diejenige Schaltstelle, die über den jeweiligen Informationsbedarf situativ entscheiden muß. Denn für das Steuerungspersonal bleibt die EDV ein Informationsmedium, das Daten nur im vorprogrammierten Rahmen selegieren kann, ohne den situativen Informationswert von Daten erkennen und zusätzliche Selektionsentscheidungen unter pragmatischen Gesichtspunkten treffen zu können. Erst in einem langwierigen Lernprozeß erwirbt das Steuerungspersonal jene Selektionskompetenz, die notwendig ist, um in kritischen Situationen aus einer enormen Datenfülle die entscheidungsrelevanten Informationen auszuwählen. Diese situative Selektionskompetenz ist nicht programmierbar. Und solange computerisierte Steuerungssysteme nur im vorprogrammierten Rahmen, aber nicht aus Erfahrungen lernen können, bleiben sie auf das personengebundene Erfahrungswissen angewiesen (Jazbinsek 1986: 19).
Steuerungsprogramme können zwar nicht aus Erfahrung lernen. Aber sie können aus menschlicher Erfahrung verbessert und weiterentwickelt werden. Dies ist neben der unmittelbaren Steuerungsaktivität ein zweiter Verwendungszweck, dem das Erfahrungswissen der Meßwartenarbeiter zugeführt wird. Die Meßwartenarbeit schließt einen genuinen Beitrag zur Informationsgewinnung ein, der die automatische Prozeßdatenerzeugung ergänzt. Dieser Beitrag ist mit bloßem Auge nicht erkennbar, weil Informationserzeugung aus Arbeitshandeln und Informationsumsetzung in Arbeitshandeln in der Steuerungstätigkeit unmittelbar zusammenfallen. Darin unterscheidet sich die Meßwarenarbeit von den Facharbeitertätigkeiten des Maschinenbaus oder der Instandhaltung, wo Arbeitsausführung und Informationserzeugung in zwei real unterscheidbare Aktionen auseinanderfallen. Bei PPS-Systemen oder bei computergestützter Instandhaltungsplanung arbeitet das Personal einerseits seine Arbeitsaufträge ab, indem es diese in Arbeitshandeln umsetzt. Andererseits gibt das Personal jene planungswichtigen Detailinformationen, die sich erst während des Arbeitsvollzugs zu erkennen geben (Störungsdiagnose, Arbeitsdauer, unvorhersehbare Ausführungsprobleme etc.), in den Computer ein. Bei der Meßwartentätigkeit dagegen ist diese zweite Funktion bereits in ihrer Hauptaufgabe enthalten. Ihre Hauptaufgabe besteht darin, aus den Prozeßrechnersignalen bestimmte Korrektur- und Nachregelungserfordernisse herauszulesen und in Steuerungsbefehle umzusetzen. Erst im Computerprogramm werden die beiden Funktionen entkoppelt. Dies geschieht dadurch, daß jeder menschliche Eingriff in den Prozeß doppelt verarbeitet wird. Als Steuerbefehl löst er Veränderungen im Realprozeß aus und als Information wird er im System abgespeichert. Als Nebenprodukt der eigentlichen Steuerungsaufgabe werden somit Informationen über das individuelle Arbeitshandeln des Personals für spätere Spezialauswertungen protokolliert (vgl. auch Dubois 1986).

Neben der unmittelbaren Prozeßsteuerung besteht also der zweite Verwendungszweck des Erfahrungswissens der Meßwarenarbeiter darin, ihre per Terminaldialog in den Prozeßrechner eingegebenen Korrekturbefehle und die ihm entnommene Zusatzinformationen abzuspeichern. Zusammen mit den ebenfalls abgespeicherten automatischen Prozeßrechneroperationen sowie den Komplementärdaten des verfahrenstechnischen Realprozesses ergibt sich ein komplettes Protokoll des mehrstündigen Gesamtablaufs vom Anfahrbeginn 
bis zum Abschluß eines Umwandlungsvorgangs. Dieses Protokoll eröffnet unterschiedliche Auswertungsmöglichkeiten. Mit seiner Hilfe kann das Leistungsverhalten des Personals und das Zusammenspiel zwischen menschlichem Eingriff und Steuerungsprogramm analysiert werden, können wiederholte häufige Störungen im Realprozeß, aber auch Mängel und Fehlerquellen in der Steuerungssoftware aufgedeckt werden. Hier leistet das Meßwartenpersonal einen unersetzlichen Beitrag. Diesen Beitrag bezeichne ich mit Blick auf die neuen Informations- und Kommunikationstechnologien als „Algorithmisierungsbeitrag“. Um diesen Algorithmisierungsbeitrag drehen sich die weiteren Überlegungen.

Der Algorithmisierungsbeitrag stellt sich gegenüber der intelligenten Dateneingabe als eine anspruchsvollere Form des Eigenbeitrags zur Wissenserzeugung dar. Beide Formen lassen sich analytisch klar voneinander unterscheiden, wenngleich sie empirisch natürlich fließend ineinander übergehen. Die analytische Differenz, die hier zugrunde liegt, ist allgemein gesprochen die zwischen Produzieren und Rationalisieren. Dabei gilt die generelle Annahme, daß der Produzent am besten weiß, wie der Produktionsprozeß, an dem er direkt beteiligt ist, verbessert und vervollkommnet, sprich: rationalisiert werden kann. In der traditionellen Betriebsorganisation dominiert die Trennung der beiden Funktionen. Produktionsbeiträge und Rationalisierungsbeiträge werden von verschiedenen Beschäftigtengruppen erbracht, die streng gegeneinander abgeschottet sind. Auf informationstechnologischer Grundlage verschieben sich die Koordinaten der klassischen Trennung von Produktion und Rationalisierung in doppelter Hinsicht. Einerseits kommt es zu einer Entdifferenzierung, indem die Beschäftigten uno actu (Steuerungspersonal, Verwaltungssachbearbeiter) oder in zwei Arbeitshandlungen (Instandhalter, Maschinenbaufacharbeiter) ihren Produktionsbeitrag leisten und zugleich Rationalisierungsinformationen bereitstellen. Andererseits kommt es zur Ausdifferenzierung des Rationalisierungsbeitrags in intelligente Dateneingabe und Algorithmisierungsbeitrag. Ich möchte nun plausibel machen, daß es einen inneren Zusammenhang zwischen intelligenter Dateneingabe und Algorithmisierungsbeitrag gibt. Dieser geht gleichsam immanent aus jener hervor. Die Plausibilität dieses inneren Zusam- menhangs ist für den Fortgang der weiteren Argumentation von zentraler Bedeutung.

Der Algorithmisierungsbeitrag des Steuerungspersonals ist keimförmig in der situativen Problembewältigung angelegt. Indem die Meßwartenarbeiter ihr Problemlösungsverhalten kreativ variieren und in ähnlichen Situationen auf bewährte Bewältigungsmuster zurückgreifen, häufen sie nach und nach einen Fundus an prozeßspezifischem und programmspezifischem Erfahrungswissen an, der ihnen einen souveränen Umgang mit der Anlage erlaubt. Im Programmprotokoll liest sich dieser Lernprozeß auf der Zeitachse möglicherweise als Abnahme menschlicher Steuerungsaktivität. Das hat natürlich nichts mit nachlassendem Eifer zu tun. Vielmehr handelt es sich um das schlichte Ergebnis allmählich gewachsener Heuristiken. Diese versetzen das Personal in die Lage, aufwendige Entscheidungsstrategien intuitiv aber treffsicher abzukürzen. Das Protokoll enthält also zahllose indirekte Hinweise auf Verbesserungsmöglichkeiten, die in den Ingenieurbüros per quantitativer Analyse zwar eingekreist, aber nicht unbedingt auch schon verstanden werden können. Dazu bedarf es der Rücksprache mit der Meßwartenmannschaft und dies ist zugleich der mögliche Ausgangspunkt eines kollektiven Reflexionsprozesses.

Ähnliches gilt für zahlreiche ,indirekt produktive" Beschäftigtengruppen aus dem gewerblichen und aus dem Angestelltenbereich wie beispielsweise Instandhaltungsfachkräfte, Materialdisponenten, Verwaltungssachbearbeiter und Konstrukteure. Ihr Algorithmisierungsbeitrag beginnt damit, daß sie Arbeitserfahrungen mit den praktischen Schwierigkeiten im Mensch-Maschine-Dialog, bei der Dateneingabe und -ausgabe, machen. Dabei werden sie auf mangelhafte Datenstrukturen, Klassifikationssysteme und unzureichende Kodierungskonventionen sowie auf Programmfehler aufmerksam. Diese Beobachtungen tauschen sie gesprächsweise mit Kollegen und Vorgesetzten aus. Sobald solche Hinweise in den realen Rationalisierungs- und Innovationsproze $\$$ durch direkte Beteiligung der Fachkräfte vor Ort einfließen, ist der Übergang zum Algorithmisierungsbeitrag vollzogen.

Unter dem Druck der „Softwarekrise“ gewinnt dieser Algorithmisierungsbeitrag gegenwärtig eine wachsende empirische Bedeutung. Informationskompetenz wird den Beschäftigten also nicht nur bei der Dateneingabe im computerisierten Dialog abverlangt, sondern in zunehmendem Maße auch bei der Beteiligung an der Softwareentwicklung. 
Die entscheidende Quelle der Belehrung ist in beiden Fällen die Diskrepanzerfahrung zwischen Alltagssprache und informatisiertem Code, zwischen überschüssigem Randwissen und verengten Kerninformationen, zwischen Realprozeß und Computerprogramm. Diese Diskrepanzerfahrung versetzt den fachkompetenten Benutzer keineswegs nur in ehrfürchtiges Staunen vor den Fähigkeiten der Computertechnologie. Denn sie zeigt ihm in schonungsloser Offenheit, was der Computer nicht kann.

Das Erfahrungswissen, welches vom Fachpersonal bei der Eingabe und Entnahme von Informationen am Terminal erworben wird, stellt sich für die Informations- und Steuerungstechnologien als eine Lebenshilfe par excellence dar. Ohne die vielen kompensatorischen Aktionen erfahrener Fachkräfte wäre kein Computerprogramm im Alltagsbetrieb wirklich lebensfähig, selbst wenn es technisch einwandfrei funktionieren sollte. Nichts anderes gilt für den Prozeß der Programmentwicklung. Je diffiziler der im Programm nachzubildende Realprozeß ist, desto notwendiger wird es für Systemanalytiker und Organisationsprogrammierer, aufs Engste mit denjenigen zu kooperieren, die über das vorinformatische Erfahrungswissen des entsprechenden Realprozesses verfügen.

Idealtypisch läßt sich der Algorithmisierungsbeitrag am Beispiel der Entwicklung sogenannter Expertensysteme verdeutlichen. Feigenbaum und McCorduck (1983) haben den dafür erforderlichen Eigenbeitrag des menschlichen Experten prägnant beschrieben. Bei der Entwicklung von Expertensystemen, wie sie seit einigen Jahren unter dem prätentiösen Etikett der „,künstlichen Intelligenz“ vorangetrieben werden, türmen sich die Schwierigkeiten vor der "notwendig zu lösenden Aufgabe, dem Menschenkopf Expertenwissen überhaupt erst $\mathrm{zu}$ entlocken und es in geeigneter Form in einer Wissensbasis zu vergegenständlichen (,knowledge acquisition'). Der ,Knowledge Engineer', dem diese zweifelhafte Aufgabe zufällt, entbehrt ironischerweise selber des relevanten Expertenwissens und soll gleichwohl den Wissensträgern Regeln entziehen, die diesen gar nicht bewußt sind. Es ist nicht böser Wille, der sie daran hindert, ihr Wissen preiszugeben, sondern der Umstand, daß sie darüber nicht in Form expliziter Regeln verfügen." (Brödner 1985: 109) Bei hochkomplexer Expertenarbeit besteht also ein enormes Risiko, daß wesentliche Parameter übersehen oder in fehlerhafte Algorithmen überführt werden. Das hat fatale Diskrepanzen zwischen Modell und Realität zur
Folge, die, wenn sie erst nachträglich entdeckt werden, irreparabel sind. Die Verständigung zwischen Experte und Wissensingenieur wird damit zum kritischen Engpaß der Informatisierung. Volperts Bemerkung, daß der Wissensingenieur „mehr als der alte Taylor auf die Mitarbeit von Experten angewiesen “ ist, um die Spezialkenntnisse aus ihren Köpfen herauszuholen (Volpert 1985: 32 ), ist deshalb eine glatte Untertreibung.

Kaum weniger kritisch stellt sich die Rückkehr des informatisierten Expertenwissens zum Benutzer dar, in den sich der Experte inzwischen verwandelt hat. Denn das in Wissensbanken und Expertensystemen objektivierte Expertenwissen ist in standardisiertes und formalisiertes Planungswissen transformiert worden, das ,nur noch schwer oder gar nicht mehr in praktiziertes Wissen, in Können umsetzbar" ist (Volpert 1985: 109). Die Pointe dieses von Volpert hervorgehobenen Sachverhalts besteht darin, daß nur der Experte selbst mit dem Expertensystem etwas anfangen kann. Denn auch für algorithmisch adäquat beschriebene Teilschritte der Expertenarbeit gilt, daß sie fachsprachliche Artefakte am Bildschirm hervorbringen, die ohne pragmatische Rückübersetzung in den situativen Entscheidungskontext völlig nutzlos sind.

Im informatisierten Produktionsproze $\beta$ erweisen sich also die erste und die dritte Kreislaufphase der Wissenstransformation, die Enteignung und die Rückkehr des Wissens, als besonders kritisch. Die systemanalytische Durchdringung des Erfahrungswissens sowie der Implementationsproze $\beta$, in welchem das entfaltete Planungswissen in Gestalt neuer Steuerungs- und Informationssysteme in den Betrieb zurückkehrt, stellen sich als hochriskante Momente der Systementwicklung dar. Dabei sind die Fehler der ersten Transformationsphase besonders tückisch. Denn was zu Beginn eines Entwicklungsprojekts falsch gemacht oder versäumt wurde, das tritt oftmals erst im Implementationsprozeß zutage. Insofern ist der Implementationsprozeß die dramatischere Phase im Innovationszyklus. In den Geburtswehen von Testlauf, Erprobung und Inbetriebnahme muß sich zeigen, ob ein System lebensfähig ist oder nicht. Eine Unzahl gescheiterter, abgebrochener und versandeter Informatisierungsprojekte belegt diese Risiken. Allerdings sind es nicht allein die Fehler unzureichender Modellbildungen, versäumte Konsultationen mit den Fachleuten vor Ort, also mangelnde ErschlieBung von Erfahrungswissen in der Frühphase der Systementwicklung, die zum Stolperstein werden. Denn auch nach gelungener Algorithmisierung 
scheitern neue Informations- und Steuerungssysteme nicht selten am Benutzerverhalten während und nach der Inbetriebnahme. Ein Unternehmen, das informatisieren will, kommt ohne das Erfahrungswissen vor Ort nicht aus. Es muß nicht nur den Widerstand der betrieblichen Fachkräfte ausräumen, , ,sondern (es) benötigt ihre positiven Beiträge: ihre ausdrückliche Ergänzungshilfe. $\mathrm{Ob}$ man sie gibt oder nicht, ist - in Grenzen - offen und an Bedingungen knüpfbar." (Ortmann 1984: 73)

Es ist sonnenklar, da $\beta$ die Informatisierung des betrieblichen Erfahrungswissens höchst verwundbar ist und insofern ein eigentümliches Politikpotential erzeugt. Dieses Politikpotential hat aber auch einen höchst gewöhnlichen Aspekt. Denn die Informatisierung spielt sich im Kontext vorgängiger Machtasymmetrie ab, die durch die Lohnabhängigkeit der Betriebsexperten konstituiert wird. Die Fachleute aus Produktion und Verwaltung agieren nicht im Elfenbeinturm, sondern sie unterliegen grundsätzlich den leistungswirtschaftlichen Zielsetzungen des Managements. Neben der Einbindung des Wissens heißt das immer auch Verdichtung, Substitution und Vernichtung von Expertenarbeit, sobald die Ökonomisierungseffekte der Expertensysteme, der Planungs- und Entscheidungstechnologien zu greifen beginnen (Weißbach 1987). Klar ist aber auch, daß eine hart durchgezogene Intensivierungs- und Substitutionsstrategie jene „Ergänzungshilfe“ aufs Spiel setzt, ohne die die Informatisierung mißlingen muß. Aus dieser Zwickmühle führt kein Weg heraus. Für Kontrahenten und Interessenten gilt es daher, sich mit und in den Paradoxien der Informatisierung auf Dauer einzurichten.

Angesichts dieser widerspruchsvollen Entwicklungsperspektive von einer „Taylorisierung der Expertenarbeit" (Volpert 1985: 43) zu reden, ist schlicht unplausibel. Volperts Behauptung, daß die Folgen der Informatisierung für die Kopfarbeit den Folgen des alten Taylorismus für die Handarbeit prinzipiell gleichen (1985: 36ff.), ist ebenso unbefriedigend wie Brödners Diagnose, wonach die Kopfarbeit „nach bewährtem Muster . . . genau den gleichen Methoden der Vergegenständlichung ihres Wissens und Könnens" unterworfen werde wie zuvor schon die Handwerkerarbeit (Brödner 1985: 78). Diese hemdsärmelige Gleichsetzung ist um so bedauerlicher, als beide Autoren immer wieder ausdrücklich auf empirische Phänomene einer eigenaktiven Informationskompetenz hinweisen, auf die Notwendigkeit einer auf Selbstbeobachtung basierenden Wissenserzeugung, die erst durch die Informatisierung ihre besondere Gestalt erhält.

\section{VI}

In der widersprüchlichen Entwicklungsperspektive der Informatisierung ist es keineswegs zufällig, da $B$ mikroelektronische Informationstechnologien und partizipative Organisationsphilosophien gleichzeitig zu konjunktureller Blüte gelangt sind. Während die Informatisierung immer systematischer auf das Erfahrungswissen zugreift und dabei mehr denn je auf den aktiven Beitrag der Beschäftigten angewiesen ist, wird sie von den neuen Partizipationskonzepten des Managements flankiert. Partizipationskonzepte verfolgen objektiv den Zweck, Eigenmotivation und kreative Ressourcen der Arbeitskräfte zu mobilisieren und für die systematische Wissenstransformation zu erschließen. So bildet sich eine spezifische Komplementaritätsbeziehung zwischen informationstechnologischer und partizipativer Informationsgewinnung heraus. Der Mensch-Maschine-Dialog tritt also keineswegs ersatzlos an die Stelle gesprächsförmiger Kommunikation, Wissenserzeugung und Reflexion. Die informationstechnologische Instrumentalisierung des Erfahrungswissens scheint geradezu eine gegenläufige Entwicklung auszulösen, die in Qualitätszirkeln, Problemlösungsgruppen und Teamkonzepten einen empirischen Niederschlag findet. Diese gegenläufige Entwicklung könnte man mit Habermas (1981) als „kommunikative Rationalisierung“ computergestützter Informations- und Planungssysteme interpretieren.

Habermas hat den Geltungsanspruch des Begriffs kommunikativer Rationalisierung, der die soziokulturelle Herausbildung einer zweckfreien Verständigungsorientierung bezeichnet, indessen ausdrücklich auf die lebensweltlichen Bereiche der Nicht-Arbeit begrenzen wollen. In der Perspektive seiner "Theorie des kommunikativen Handelns" käme die Informatisierung ähnlich wie bei Weizenbaum nur als imperialistische Bedrohung einer die Lebenswelt zersetzenden „systemischen Rationalisierung" ins Blickfeld. Johannes Berger (1982) hat diese verengte Perspektive mit guten Gründen bestritten. Er beharrt in Umkehrung zur Habermasschen Kolonialisierungsthese auf der Möglichkeit, daß lebensweltliche, „grüne“ Handlungsorientierungen von außen in den ,,industriell-bürokratischen Komplex" eindringen könnten. Ich möchte hier noch einen Schritt weitergehen und zeigen, daß die „systemische“ Informatisierung des 
betrieblichen Produktionsprozesses gleichsam von innen heraus neue Formen einer ,kommunikativen" Verständigungsorientierung hervorbringt. Dateneingabe und Algorithmisierungsbeitrag stellen sich im Informatisierungskontext als hochproblematische Leistungen dar, und ich vermute, daß in diese Problematik zugleich die entwicklungslogische Möglichkeit ihrer kommunikativen Rationalisierung eingeschlossen ist.

Jazbinsek (1986: 26f) hat unter Rückbezug auf eine Argumentationsfigur Luhmanns (1984) die These vertreten, daß die informationstechnologische Vermittlung von Kommunikationsprozessen den Konformitätszwang gegenüber den Benutzern erhöht und gerade deswegen „Irrationalitäten“ bei der Dateneingabe provoziert. Sobald die direkte menschliche Kommunikation auf maschinenvermittelte Kommunikationssysteme umgestellt wird, wird der Verbindlichkeitsgrad jeder einzelnen Mitteilung auf das Hochplateau definitiver Festlegung angehoben, auf dem informelle Abstimmungs- und Klärungsprozesse ausgeschaltet sind. Nonkonformistische Handlungsalternativen können nicht mehr probeweise vorgebracht werden, da die sonst üblichen Rückzugsmöglichkeiten gesprächsförmiger Kommunikation verbaut sind. Unter den Bedingungen einer informationstechnologischen Pseudoöffentlichkeit ist jede einmal ins Informationssystem gelangte Mitteilung definitiv, jede Entscheidung endgültig. ,Der Informant muß jetzt allein verantworten, was er mitteilt", und so orientiert er sich im Zweifel am Modus der „erwartungskonformen Irrationalität" und nicht am Modus des „rationalen Nonkonformismus“. Für den Informationsempfänger und im weiteren Sinne für den Proze $B$ der Datenverarbeitung verschärft sich damit das Problem, eine Mitteilung als richtig und wichtig zu verstehen und zu akzeptieren. Hier drohen Gefahren, die im Extremfall ein Informationssystem ruinieren können.

Ob das Management trotz solcher Risikoerfahrungen an der .,imperialistischen“ Fiktion festhält, mit Hilfe informationstechnologischer Systeme sämtliche Kontrollücken schließen zu können, oder ob es die Risiken des EDV-Einsatzes mit neuen partizipativen Leitbildern beantwortet: In beiden Entwürfen reflektiert sich eine funktionelle Differenzierung zwischen gesprächsförmiger und computervermittelter Kommunikationsweise, in welcher ihre Verschiedenheit ebenso wie ihre unauflösliche Verschränkung ins Bewußtsein der Akteure tritt. „Infolge der Informatisierung bekommt der Dialog per Computer die Funktion, Standardthemen zu behandeln. Dagegen ist es die Funktion der (formellen oder informellen) Gespräche, Unvorhergesehenes und Riskantes zu thematisieren (und) rationalen Nonkonformismus zu artikulieren." (Jazbinsek 1986: 32). Je nach Leitvorstellung werden die gesprächsförmigen „Denk- und Kommunikationskulturen" (Seltz/Hildebrandt 1985: 106f.) entweder der Informalität und dem Zufall überlassen oder in formelle Besprechungskreise, Problemlösungsgruppen etc. überführt und dort gezielt kultiviert und weiterentwickelt. Die neuen Organisationsphilosophien des Organization Development und der Unternehmenskultur lassen sich insofern auch als das Bemühen des Managements interpretieren, die kontraproduktiven Effekte der informationstechnologischen Festlegung durch partizipative Verständigungsformen zu beheben und die ohnehin vorhandenen Entscheidungs- und Kommunikationschancen des Personals im Unternehmensinteresse zu kanalisieren.

Im Hinblick auf den Algorithmisierungsbeitrag treten Differenz und reale Verschränkung beider Kommunikationsweisen noch artikulierter in Erscheinung. Im Entwicklungsprozeß von EDV-Systemen springt die praktische Notwendigkeit, Algorithmen im Medium einer kommunikativen Rationalisierung zu erarbeiten, geradezu ins Auge. Auch hier kommt der Entwicklung von Expertensystemen wiederum exemplarische Bedeutung $\mathrm{zu}$. Feigenbaum und McCorduck (1983: 77) haben die Aufgabe der Wissensingenieure, ein ihnen fremdes Expertenwissen in Algorithmen umzusetzen, in folgende Metapher gekleidet: Das heuristische Wissen der Experten müsse „mühsam aus ihren Köpfen ausgegraben werden, Juwel für Juwel. Die Bergleute nennt man Wissensingenieure."

Die Bergbaumetapher fängt nun aber das, worauf es ankommt, gerade nicht ein: daß es sich um einen interaktiven Verständigungsproze $B$ handelt, in welchem das heuristische Wissen des Experten beiden Partnern, sowohl dem Experten als auch dem Wissensingenieur, zu Bewußtsein kommt. Feigenbaum und McCorduck unterliegen hier dem typischen Selbstmißverständnis eines instrumentalistisch halbierten Rationalitätsbegriffs, indem sie die Entwicklung von Expertensystemen nach dem Modell der Gegenstandsbearbeitung zu begreifen suchen. Verständigung zwischen Kommunikationspartnern bedeutet jedoch, daß Experte und Wissensingenieur sich wechselseitig in die Explorations- und Rationalitätsmuster des je anderen hineindenken. Der Informatiker in seiner Rolle als Wissensingenieur kommt dabei gar nicht umhin, 
seine algorithmischen Modellbildungen im offenen Dialog zu hinterfragen und hinterfragen zu lassen. Die algorithmischen Verkürzungen seines Systemmodells müssen vom Experten als künftigem Benutzer kritisch gegengelesen werden. Denn ein Expertensystem, das nicht selbst wiederum durch die Kritik des betrieblichen Fachmanns, der einmal damit arbeiten soll, hindurchgegangen ist, wird seine praktische Bewährungsprobe kaum bestehen können.

Aufklärung ist im dialogischen Prozeß der Systementwicklung also keine Einbahnstraße. Ein Wissensingenieur oder Systemanalytiker, der sich allein darauf beschränken wollte, die naiven Alltagsheuristiken des betrieblichen Fachmanns algorithmisch zu explizieren, ohne zugleich auch etwas über die Erkenntnisgrenzen seiner informatorischen Naivität und seiner eigenen systemanalytischen Alltagsheuristiken zu lernen, wird seine Aufgabe verfehlen. Soll die Systementwicklung gelingen, wird sich der Wissensingenieur nicht bloß als Bergmann verstehen und damit „rationalistisch" mißverstehen, sondern er wird sich als sokratischer Mäeutiker betätigen. Im praktischen Diskurs mit dem Experten wird er die sokratische Hebammenkunst, die Mäeutik, ausüben und die im Partner schlummernden intuitiven Erkenntnisse durch geschicktes Fragen bewußt machen.

Systemanalyse und Algorithmisierung von Expertenwissen setzen überschüssige Aufklärungsprozesse frei, die nicht zuletzt den Systemanalytiker über die Grenzen der informatorischen Modellbildung belehren. Im mäeutischen Dialog wird deutlich, daß Erfahrungswissen und Computerprogramm, Heuristik und Algorithmus sich nicht wie implizites und explizites Wissen zueinander verhalten. Intuitives Erfahrungswissen kann bewußt gemacht und expliziert werden, ohne in Algorithmen überführt zu werden, und Algorithmen müssen im Horizont des Erfahrungswissens verstanden und kontextspezifisch interpretiert werden. Die kommunikative Bewußtwerdung verschiedenartiger Rationalitätsmuster bleibt freilich eine flüchtige Erscheinung, die sich am fertigen Expertensystem nur noch unvollständig ablesen läßt. Aber, und auch das gilt es festzuhalten, es handelt sich um ein Bewegungsmoment, das im Zuge der Informatisierung beständig reproduziert wird.

\section{VII}

Wenn meine These zutrifft, so sind instrumentellfunktionalistische Rationalisierung und reflexiv- kommunikative Rationalisierung weder im strengen Habermasschen Sinn als Ausschließungsverhältnis bestimmbar, noch lassen sie sich mit Luhmann als komplexitätsbedingtes Steigerungsverhältnis beschreiben. Vielmehr setzt die Informatisierung als ein besonderer Modus der instrumentellen Rationalisierung verständigungsorientierte Kommunikationsformen frei, die sich gegen Interpretationen, wie sie vom Imperialismustheorem vorgebracht werden, prinzipiell sperren. Denn das Imperialismustheorem ist blind für die beharrliche Reproduktion des kommunikativen Eigensinns, der im elektronischen Gehäuse sein offensichtlich höchst lebendiges Unwesen treibt. Die Unausrottbarkeit jenes Eigensinns ist jedoch kein Grund für gesellschaftstheoretischen Optimismus. Eine $\mathrm{Zu}$ kunft der Lohnarbeit im Zeichen von selbstbestimmter Autonomie oder .,projektförmiger Selbstorganisation" (Krohn/Rammert 1984) ist nicht deshalb schon angesagt, weil der Mythos vom Imperialismus der instrumentellen Vernunft entmythologisierbar ist.

Die Diagnose, daß der Algorithmisierungsbeitrag des betrieblichen Fachmanns wirksam nur im Medium kommunikativer Verständigung erschlossen werden kann, ist also nicht emphatisch gemeint. Sie besagt zunächst lediglich, daß der informationstechnologische Entropietod, von der sozialphilosophischen Instrumentalismuskritik seit Jahr und Tag beschworen, nicht eintreten wird. Aber das hat mit einer Verwirklichung des spiegelverkehrten Ideals einer herrschaftsfreien Produktions- und Diskussionsgemeinschaft unmittelbar nichts zu tun. Jene algorithmische Aufklärung spielt sich nämlich im interessengeladenen Spannungsfeld von Verwertungs- und Herrschaftsbeziehungen $a b$, die immer wieder geräuschvoll durch die Verständigungsorientierung der Dialogpartner hindurchgreifen. Denn Softwareingenieure und betriebliche Fachleute kooperieren in Projekten, deren Ziele von außen gesetzt werden und deren Handlungsraum durch strikte Effizienz- und Kostenkontrollen begrenzt ist. Vor diesem Hintergrund bleibt Wissenserzeugung, und sei sie noch so tief im Medium der kommunikativen Selbstbeobachtung verwurzelt, stets als das erfahrbar, was sie ebenfalls ist: Wissensenteignung. Hier wird es immer wieder Vorkommnisse (Vallée 1984: 115ff.) geben, die eine gespenstische Szenerie schlaglichtartig erhellen, in welcher der Wissensvampir im Kostüm der Hebamme auftritt.

Als soziologischer Beobachter hat man nüchtern zu konstatieren, daß das neue Leitbild des mäeuti- 
schen Systemanalytikers ebenso wie das alte Leitbild des tayloristischen Zeitnehmers zu einer kapitalistischen Entwicklungsdynamik gehört, die im Widerstreit zwischen Unternehmensplanung und Marktanarchie, Innovation und Verkrustung, Herrschaftsanspruch und Partizipationsangebot zerrissen ist. Diese Widersprüche lassen sich mit den eindimensionalen Kategorien des Imperialismustheorems begrifflich nicht einfangen. Solche Kategorien taugen bestenfalls zur Selbsteinschüchterung. Resignation oder gar Fatalismus sind jedoch völlig fehl am Platze, weil die Informatisierung ihre eigenen Entwicklungswidersprüche hervortreibt und eben dadurch Alternativen schafft und Optionen eröffnet.

\section{Literatur}

Berger, J., 1982: Die Versprachlichung des Sakralen und die Entsprachlichung der Ökonomie. Zeitschrift für Soziologie 4: 353-365.

Böhme, G./van den Daele, W./Krohn, W., 1972: Die Finalisierung der Wissenschaft. Zeitschrift für Soziologie 2: $128-144$.

Braverman, H., 1978: Die Arbeit im modernen ProduktionsprozeB. Frankfurt.

Briefs, U., 1980: Arbeiten ohne Sinn und Perspektive? Gewerkschaften und „Neue Technologien“. Köln.

Brödner, P., 1985: Fabrik 2000. Alternative Entwicklungspfade in die Zukunft der Fabrik. Berlin: Sigma.

Dubois, P. 1986: Travail et Nouvelles Technologies: Un Essai de Synthese. Unveröffentlichter Beitrag zum Internationalen Kolloquium der Arbeitssoziologie. Bologna.

Feigenbaum, E. A./McCorduck, P., 1983: The Fifth Generation. Reading.

Habermas, J., 1981: Theorie des kommunikativen Handelns (2 Bde.). Frankfurt: Suhrkamp.

Hack, L./Hack, I., 1985: Die Wirklichkeit, die Wissen schafft. Zum wechselseitigen Begründungsverhältnis von "Verwissenschaftlichung der Industrie“" und .,Industrialisierung der Wissenschaft". Frankfurt/New York: Campus.

Horkheimer, M./Adorno, Th. W., 1947: Dialektik der Aufklärung. Philosophische Fragmente. Amsterdam: Querido.

Institut für Sozialforschung (Hrsg.), 1981: Gesellschaftliche Arbeit und Rationalisierung. Neuere Studien aus dem Institut für Sozialforschung in Frankfurt am Main. Leviathan Sonderheft 4.

Jazbinsek, D., 1986: Vom blauen zum weißen Kragen. Die Informatisierung der Fabrik und ihre Folgen für die Industriearbeit. Unveröffentlichtes Manuskript. Bielefeld.

Karlsen, T./Oppen. M., 1986: Fachqualifikationen und die Grenzen der Verwaltungsautomation. Unveröffentlichtes Manuskript, WZB. Berlin.
Koch, C., 1983: Jenseits der Gesellschaft. Die Zukunft im elektronischen Gehäuse. Merkur 37: 737-746.

Krohn, W./Rammert, W., 1984: Technologieentwicklung: Autonomer Prozeß und industrielle Strategie. S. 411-433 in: B. Lutz (Hrsg.), Verhandlungen des 22. Deutschen Soziologentags in Dortmund. Frankfurt New York: Campus.

Kubicek, H./Rolf, A., 1985: Mikropolis. Mit Computernetzen in die "Informationsgesellschaft". Hamburg: VSA.

Luhmann, N., 1984: Soziologische Aspekte des Entscheidungsverhaltens. Die Betriebswirtschaft 4: 591-603.

Malsch, Th., 1983: Erfahrungswissen versus Planungswissen. Facharbeiterkompetenz und informationstechnologische Kontrolle am Beispiel der industriellen Instandhaltung. S. 231-251 in: U. Jürgens/F. Naschold (Hrsg.), Arbeitspolitik, Leviathan Sonderheft 5.

Malsch, Th./Seltz, R., 1987: Zur Einführung: Die aktuelle Diskussion über die Entwicklung neuer Produktions- und Rationalisierungsmodelle. S. 11-34 in: 'Th. Malsch/R. Seltz (Hrsg.), Die neuen Produktionskonzepte auf dem Prüfstand. Beiträge zur Entwicklung der Industriearbeit. Berlin: Sigma.

Manske, F./Wobbe, W., 1984: Rechnerunterstützte Sy steme der Fertigungssteuerung in der Kleinserienfertigung - Auswirkungen auf die Arbeitssituation und Ansatzpunkte für eine menschengerechte Arbeitsgestaltung. Göttingen.

Mill, U., 1986: Organisation als Sozialsystem. Ein Kommentar. S. 199-218 in: R. Seltz/U. Mill/E. Hildebrandt (Hrsg.), Organisation als soziales System. Kontrolle und Kommunikationstechnologie in Arbeitsorganisationen. Berlin: Sigma.

Ortmann, G., 1984: Der zwingende Blick. Personalinformationssysteme - Architektur der Disziplin. Frankfurt/ New York: Campus.

Polanyi, M., 1985: Implizites Wissen. Frankfurt: Suhrkamp.

Schmiede, R., 1983: Abstrakte Arbeit und Automation Zum Verhältnis von Industriesoziologie und Gesellschaftstheorie. Leviathan 1: 55-78.

Schmiede, R./v. Greiff, B., 1985: Industriesoziologie als positive Geschichtsphilosophie? Über die „Trendwen-

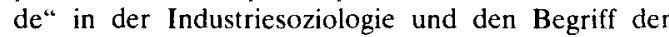
"reellen Subsumtion". Leviathan 2: 291-306.

Seltz, R./Hildebrandt, E., 1985: Produktion, Politik und Kontrolle. S. 91-124 in: F. Naschold (Hrsg.), Arbeit und Politik. Gesellschaftliche Regulierung der Arbeit und der sozialen Sicherung. Frankfurt/New York: Campus.

Sohn-Rethel, A., 1970: Geistige und körperliche Arbeit. Zur Theorie der gesellschaftlichen Synthesis. Frankfurt: Suhrkamp.

Sorge, A., 1985: Informationstechnik und Arbeit im sozialen Prozeß. Arbeitsorganisation, Qualifikation und Produktivkraftentwicklung. Frankfurt: Campus.

Turkle, S., 1984: Die Wunschmaschine. Vom Entstehen der Computerkultur. Reinbek: Rowohlt.

Vallée, J., 1984: Computer Netze. Träume und Alpträume von einer neuen Welt. Reinbek: Rowohlt. 
Veltz, P., 1986: Informatisation des industries manufacturières et intellectualisation de la production. Sociologie du Travial 1: 5-22.

Volpert, W., 1985: Zauberlehrlinge. Die gefährliche Liebe zum Computer. Weinheim/Basel: Beltz.

Weißbach, H.-J., 1987: Wissenseinbindung oder Wissensvernichtung? Zur Problematik von Technologieprognosen am Beispiel der Expertensysteme. Erscheint in: Jahrbuch Technik Nordrhein Westfalen 1987. Bonn.
Weizenbaum, J., 1978: Die Macht der Computer und die Ohnmacht der Vernunft. Frankfurt: Suhrkamp.

Wood, St., 1986: Neue Technologien, Arbeitsorganisation und Qualifikation. Die britische Labour-ProcessDebate. Prokla 62: 74-104. 